\title{
The failure criterion based on hydrogen distribution ahead of the fatigue crack tip
}

\author{
Yu. G. Matvienko \\ Mechanical Engineering Research Institute of the Russian Academy of Sciences, 4 M. Kharitonievsky Per., 101990 Moscow, \\ Russia \\ matvienko7@yahoo.com
}

\begin{abstract}
The hydrogen effect on the fracture toughness and fatigue crack growth behaviour in the martensitic high strength steel is investigated. The secondary ion mass spectrometry method has been employed to analyse the distribution of hydrogen concentration in the zone of the crack tip and at its edges. Changes in hydrogen concentration are observed in the vicinity of the propagating crack tip and at a remote site. The hydrogen peak $C_{H}$ is reduced and moves away from the fatigue crack tip with the increase of the maximum stress intensity factor $K_{\max }$. The concept of damage evolution is used to explain fatigue crack propagation in connection with the hydrogen redistribution ahead of the crack tip. The physical failure criterion based on the hydrogen peak in the vicinity of the fatigue crack tip and the maximum stress intensity factor has been proposed. The criterion reflects changes in the hydrogen peak which resulted from the hydrogen redistribution due to the increase of the maximum stress intensity factor as the crack length increases under fatigue loading.
\end{abstract}

KEYWORDS. Hydrogen distribution ahead of the crack tip; Fatigue crack growth; SIMS; Local failure criterion; High strength steel.

\section{INTRODUCTION}

$\mathrm{R}$ ecently many works have been performed on the hydrogen embrittlement of high strength steels (e.g., [1-4]). The deleterious effects of hydrogen on the mechanical properties of high strength martensitic steels are known to have caused premature failures. For example, it was found that the threshold stress intensity factor in steels decreases drastically in response to increased dissolved hydrogen concentration (e.g., $[2,5,6])$. Although it has been reported that hydrogen degrades mechanical properties of metallic materials, there have been few studies on the effect of hydrogen on fatigue behaviour ([7-9]).

It has been known that the fracture initiates in region of highly localized stress in which hydrogen is concentrated as a result of an augmented diffusion of hydrogen. To explain this phenomenon in the case of monotonic loading, a diffusion model was proposed by Liu [10] and developed by Kim et al. [3] introducing a fracture criterion as the critical hydrogen concentration at a critical distance ahead of the crack tip. However, until recently only a few papers [11-13] have reported on the experimental distribution of hydrogen ahead of a crack tip. Under monotonic mixed (I/II) mode loading there are two hydrogen accumulation peaks ahead of the crack tip [13], which correspond to the maximum hydrostatic stress and the maximum equivalent plastic strain, respectively. The experimental results also revealed that hydrogen distribution in the vicinity of the fatigue crack tip is related to the stress-strain fields surrounding the crack tip [12,14]. It should be also 
noted that the most essential results on the numerical analysis of the effect of cyclic loading on hydrogen diffusion and concentration around a crack tip was published by A.T. Yokobori et al. [15].

In this research, experimental analysis of the distribution of hydrogen concentration ahead of the crack tip in the martensitic high strength steel under hydrogen induced cracking and fatigue I mode loading conditions has been carried out. The generalized concept of damage evolution has been employed to explain fatigue crack propagation in connection with the hydrogen redistribution ahead of the crack tip. The physical criterion of local failure based on the hydrogen peak in the fracture process zone and the maximum stress intensity factor has been suggested.

\section{EXPERIMENTAL PROCEDURES}

$\mathrm{T}$

he martensitic high strength steel is investigated to analyse the effect of hydrogen charge on the fracture toughness, the fatigue crack growth rate and the distribution of hydrogen concentration ahead of the crack tip. The fraction of retained austenite in the steel did not exceed $10 \%$. Chemical composition of the studied steels is given in Tab. 1. Mechanical properties of the steel at room temperature are the following: the Young's modulus E=210 $\mathrm{GPa}$, yield strength $\sigma_{y}=850 \mathrm{MPa}$ and ultimate strength $\sigma_{u}=1150 \mathrm{MPa}$.

\begin{tabular}{ccccccc}
\hline $\mathrm{C}$ & $\mathrm{Cr}$ & $\mathrm{Ni}$ & $\mathrm{Si}$ & $\mathrm{Mn}$ & $\mathrm{P}$ & $\mathrm{S}$ \\
0.06 & 16 & 7 & $<0.8$ & $<0.8$ & $<0.03$ & $<0.02$ \\
\hline
\end{tabular}

Table 1: Chemical composition of high strength steel (weight \%).

The hydrogen-charged and pre-fatigued specimens were employed. Hydrogen was artificially charged into specimens by a cathodic charging method before the tests. The solution used for the cathodic charging was a 10 mass $\% \mathrm{H}_{2} \mathrm{SO}_{4}$ aqueous solution with an addition of $\mathrm{SeO}_{2}$. The current density was $i=1 \mathrm{~A} / \mathrm{dm}^{2}$ and charging time was 5 hours. Prior to immersing the specimen in the solution, its surface (with the exception of the crack surface) was coated with a chemically stable lacquer.

The testes were carried out on HUS-1025 machine at room temperature in laboratory air. The fracture toughness $K_{C}$ was measured using compact-tension specimens $(60 \times 60 \mathrm{~mm})$ with $5 \mathrm{~mm}$ thickness according to the standard method reported in the ASTM Standard E399.

The fatigue crack growth tests were conducted in order to clarify the hydrogen effect on fatigue crack growth behaviour and the distribution of hydrogen concentration ahead of the fatigue crack tip. Rectangular specimens $50 \mathrm{~mm}$ high and 5 $\mathrm{mm}$ thick with an edge crack loaded in cantilever bending were employed. The loading frequency was $20 \mathrm{~Hz}$ with a constant amplitude sinusoidal waveform for the applied load. The stress ratio was maintained at $R=-0.3$. The length of a growing fatigue crack was recorded by an optical microscope. At the given load or fatigue crack length the specimen was unloaded and 10x10 mm templates, which included the zone of the crack tip, were cut out from it. Template sizes were caused by sizes of the analytical chamber of the mass spectrometer.

The secondary ion mass spectrometry method was then used to analyse the distribution of hydrogen in the zone of the crack tip and at its edges [12]. The small size of the analysed area $(5 \mu \mathrm{m})$ was ensured using a molybdenum diaphragm placed on the specimen. Prior to this, molybdenum was degassed in vacuum. The sensitivity of analysis for hydrogen was $10^{-2} \mathrm{~cm}^{3} / 100 \mathrm{~g}$. The reproducibility of the results of determining the intensities of the spectral lines was high. The mass spectrometric results were calibrated using reference specimens employed in installations for vacuum heating manufactured by LECO Company with linear grain sizes in the metal larger than $30 \mu \mathrm{m}$. This eliminated the apparatus error in recording local hydrogen concentrations associated with segregations of hydrogen at the grain boundary in the reference specimen since the size of the ion beam in analysis was smaller than the size of the grain within which the distribution of hydrogen was usually uniform. It should be noted that secondary ion mass spectrometry was successfully employed for an analyses of the hydrogen distribution around the fatigue crack on type 304 stainless steel [16]. According to the reported data [17], the fatigue crack growth is accompanied by microplastic deformation and formation of hydrogen collectors and traps. This greatly reduces the diffusion mobility of hydrogen in the zone of the crack tip.

The curve of hydrogen distribution through thickness for the specimen during removal of layers of the material was constructed (Fig. 1). It can be seen that the position of the maximum hydrogen concentration remains unchanged in specimens with different crack lengths. After finding the depth with the maximum concentration of hydrogen, the distribution of hydrogen ahead of the fatigue crack tip was measured at this depth. 


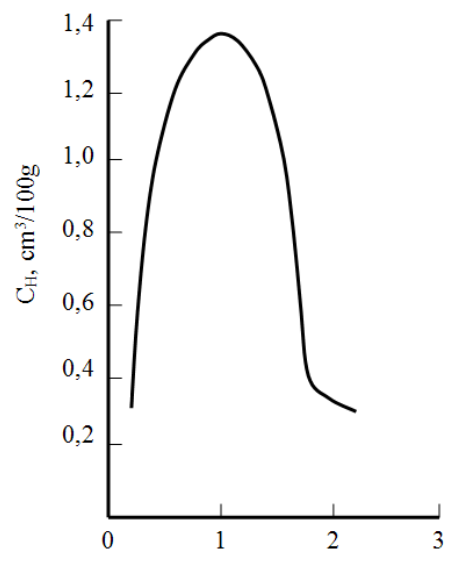

In the thickness of the specimen, $\mathrm{mm}$

Figure 1: Hydrogen distribution $C_{H}$ through the thickness for the hydrogen-charged specimen at the distance of $30 \mu m$ from the $\operatorname{crack} \operatorname{tip}\left(K_{\max }=20 M P a \sqrt{m}\right)$.

\section{HYDROGEN CHARGING CONDITIONS}

he problem of the effect of hydrogen charging conditions on the character of the hydrogen distribution in the specimen with a crack has been analysed. For this reason, the fatigue pre-cracked CT specimens were hydrogen charged in the same solution as the main set of the specimens at different values of the current density over a period of 2 hours under constant static load corresponding to two applied (constant) stress intensity factor values $K_{10}$ (Tab. 2).

\begin{tabular}{ccc}
\hline$K_{10}(\operatorname{MPa} \sqrt{m})$ & $\begin{array}{c}\text { Current density } \\
\left(\mathrm{A} / \mathrm{dm}^{2}\right)\end{array}$ & $C_{H} / C_{0}$ \\
\multirow{2}{*}{30} & 6 & 2.9 \\
& 10 & 3.0 \\
50 & 6 & 3.8 \\
& 10 & 3.8 \\
\hline
\end{tabular}

Table 2: The effect of hydrogen charging conditions on the hydrogen distribution ahead of the crack tip on crack extension line.

The distribution of hydrogen ahead of the crack tip was measured by the same procedure as for the specimens after the fatigue test [12]. The values of the ratio of the maximum local concentration $C_{H}$ of hydrogen ahead of the crack tip to the volume concentration $C_{0}$ of hydrogen in the specimen were estimated. The results clearly show that the variation of the cathodic current density in these ranges does not influence on the $C_{H} / C_{0}$ ratio which is determined only by the value of the applied stress intensity factor $K_{10}$.

\section{THE EFFECT OF HYDROGEN ON FATIGUE CRACK BEHAVIOUR}

$\Lambda$ negligible crack tip plastic zone is created due to the hydrogen embrittlement, mechanical properties of the highstrength steel and a low applied stress. It means that a linear elastic fracture mechanics methodology can be used to quantify the fracture toughness and fatigue crack growth behaviour. It follows that the maximum stress intensity factor must have an influence on fatigue damage evolution and the hydrogen distribution in the vicinity of the crack tip and, as a result, the physical growth of the fatigue crack. 


\begin{tabular}{ccccc}
\hline Material & $K_{C}(\operatorname{MPa} \sqrt{m})$ & $C\left(\frac{m / \text { gcle }}{(\operatorname{MPa} \sqrt{m})^{n}}\right)$ & $m$ & $V^{*}{\text { (micron / gycle })^{1}}^{1}$ \\
$\begin{array}{c}\text { Uncharged } \\
\begin{array}{c}\text { Hydrogen- } \\
\text { charged }\end{array}\end{array}$ & 85 & $2.53 \cdot 10^{-12}$ & 2.74 & 3.84 \\
\hline
\end{tabular}

Table 3: The fracture toughness and the fatigue crack growth rate parameters

${ }^{1 /}$ The value of $V^{*}$ corresponds to the stress intensity factor range $\Delta K$ varied from $20 \mathrm{MPa} \cdot \mathrm{m}^{1 / 2}$ to $50 \mathrm{MPa} \cdot \mathrm{m}^{1 / 2}$.

To analyse the effect of hydrogen on the fracture toughness and fatigue crack propagation, the procedure, described in Experimental procedures section for hydrogen charged specimens and tests, has been employed. Experimental results revealed that the fracture toughness $K_{C}$ and fatigue crack growth behaviour in the high-strength steel are in general dependent on the hydrogen content (Tab. 3). The fatigue crack growth rate parameters $C$ and $m$ refers to the Paris relationship. The fatigue crack growth rate $d l / d N$ versus the stress intensity factor range $\Delta K$ curve in the uncharged specimen is lower than that in the hydrogen-charged specimens (Fig. 2). The fatigue crack in the hydrogen-charged specimens propagates at the same value of $d l / d N$ as in the uncharged specimen at lower (by 30-40\%) values of $\Delta K$ in the near-threshold region. However, there is no significant difference in the fatigue fracture toughness $K_{f C}$ of the uncharged and hydrogen-charged specimens.

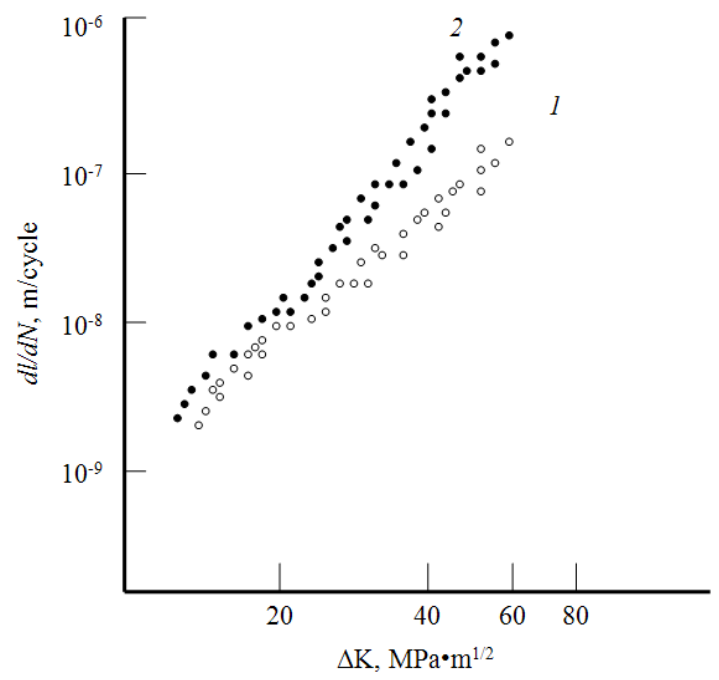

Figure 2: Fatigue crack growth behaviour: 1 - uncharged specimens, 2 - hydrogen-charged specimens

Experimental analysis of the distribution of hydrogen ahead of the crack tip under hydrogen induced cracking and fatigue I mode loading conditions has been carried out on a secondary ion mass spectroscope. The results on the distribution of hydrogen had been obtained and summarized for various periods of fatigue crack growth (or the maximum stress intensity factor) [12]. The concentration curves of hydrogen distribution in the sections normal to the crack surface and ahead of the crack tip on the crack extension line are plotted in Fig. 3 and 4, where $C_{H}$ is the local hydrogen concentration. It can be seen that there is a hydrogen accumulation peak ahead of the crack tip, which is located on some distance ahead of the crack tip (Fig. 4). Changes in the hydrogen concentration were observed in the vicinity of the propagating crack tip and at a remote site. The hydrogen peak $C_{H}$ is reduced and moves away from the crack tip as the maximum stress intensity factor $K_{\max }$ increases. The hydrogen concentration gradient also decreases. At the same time, the hydrogen concentration far away from the crack tip is increased by increasing the value of $K_{\max }$. So, the values and sites of hydrogen accumulation under fatigue loading are dependent on the magnitude of the maximum stress intensity factor. 


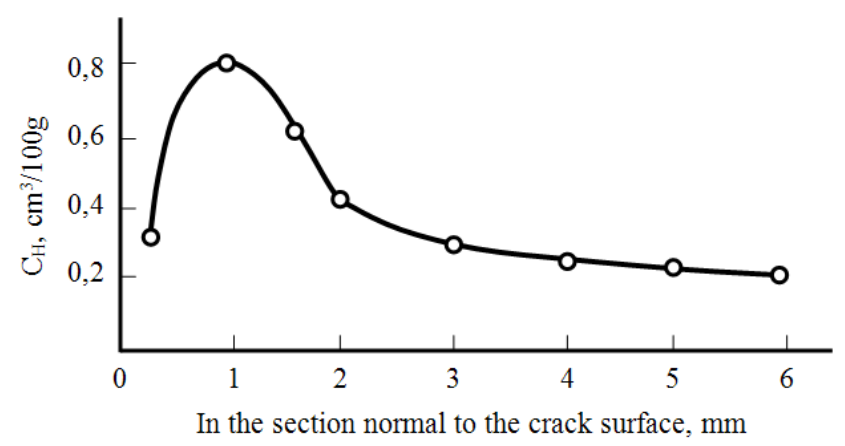

Figure 3: Hydrogen distribution $C_{H}$ in the section normal to the crack surface at a distance of $3 \mathrm{~mm}$ behind the crack tip.

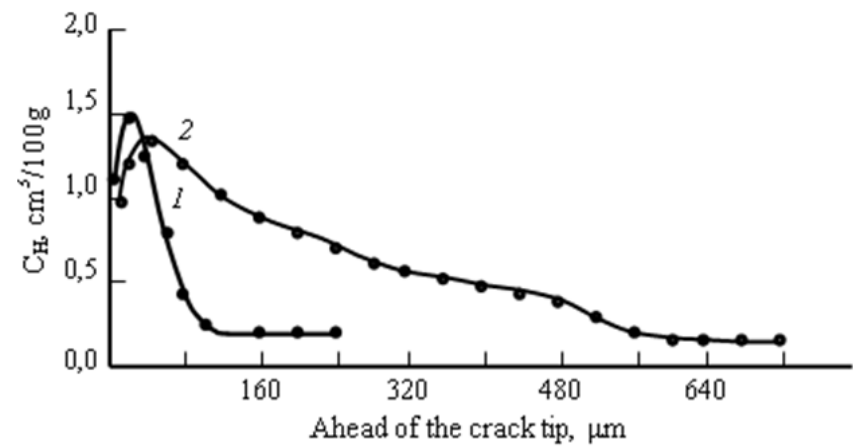

Figure 4: Hydrogen distribution $C_{H}$ ahead of the crack tip on the crack extension line: line 1 corresponds to $K_{\max }=20 \mathrm{MPa} \sqrt{m}$, line 2 corresponds to $K_{\max }=68 \mathrm{MPa} \sqrt{m}$.

The trend of the redistribution of hydrogen ahead of the propagating fatigue crack tip can be reflected on the basis of the generalized concept of damage evolution $[12,14]$.

\section{THE CONCEPT OF DAMAGE EVOLUTION}

7 he evolution approach [18] has been extended to deformation and fracture processes of a mechanical loaded system, i.e. "solid - damage". It is assumed [14] that the accumulation of damage (the system state) is determined by the scalar $0 \leq \Psi \leq 1$ which is the single state variable $q=\Psi$. The controlling parameters $\xi$ for deformation and failure processes of solids could be stress and strain, the stress intensity factor, temperature and other parameters, which are essential in the consideration of the damage accumulation process.

It is postulated that deformation and fracture processes are governed by some general functional law of damage accumulation [14]. For a simple case the damage evolution law can be formulated as

$$
\frac{d \Psi}{d \tau}=-A\left(\frac{\xi}{\Psi}\right)^{n}
$$

where $A>0, \mathrm{n} \geq 0$ are material (the "solid - damage" system) constants for the fracture process under study. The evolution law (1) can be made more precise when the physical and mechanical aspects of a failure process are more clearly understood by examining the fracture mechanisms of the solid and the type of loading under study. The value of $\Psi$ decreases with an increase of time $\tau$ during the process of the accumulation of damage in a solid. The value $\Psi=1$ corresponds to the non-damaged state of a solid when $\tau=0$, and the value $\Psi=\Psi_{c}$ corresponds to the critical state when $\tau=\tau_{c}$, where $\tau_{c}$ is the critical time. So, failure occurs in a solid if the damage reaches the critical value $\Psi=\Psi_{c}$ at $\tau=\tau_{c}$. The following relationship can be written as follows by integrating Eq. (1) from $\Psi=1$ to $\Psi=\Psi_{c}$ 


$$
\Psi_{c}^{1+n}=1-A(1+n) \int_{0}^{\tau_{c}} \xi^{n} d \tau
$$

Eq. (2) is transformed into the following equation for the determination of the critical time $\tau_{c}$

$$
\tau_{c}=\frac{1-\Psi_{c}^{1+n}}{A(1+n) \xi^{n}}
$$

if the controlling parameter $\xi$ is constant. Taking into account Eqs. (1) and (3), the cumulative damage law is expressed in the integral form

$$
\int_{0}^{\tau_{c}} \frac{d \tau}{\tau_{c}}=1
$$

The influence of the controlling parameter $\xi$ on the critical time may now be analysed for damage evolution in solids. First it is assumed that the critical value $\Psi_{c}$ is constant for the deformation and failure process under study, and the critical state of a damaged solid can be reached for various combinations of the controlling parameter and time $\tau$. It has been suggested therefore that the critical value $\Psi_{c}$ [Eq. (2)] is also reached when the controlling parameter $\xi$ is equal to the critical value $\xi_{c}$ at some fixed time (or a unit of time) $\tau=\tau^{*}<\tau_{c}$, that is

$$
\Psi_{c}^{1+n}=1-A(1+n) \xi_{c}^{n} \tau^{*}
$$

The evolution equation at $\Psi_{c}=$ const is derived from Eqs. (2) and (5), namely

$$
\int_{0}^{\tau_{c}} \xi^{n} d \tau=\xi_{c}^{n} \tau^{*}
$$

This equation may be rewritten at $\xi=$ const as

$$
\tau_{c}=\tau^{*}\left(\frac{\xi_{c}}{\xi}\right)^{n}
$$

The damage evolution equation allows one to estimate the critical time for a solid to reach its critical state under the given controlling parameter for the deformation and fracture processes being studied.

It should be noted that exponent $n$ in basic equations has different physical meaning for different physical phenomena and corresponding equations. What is why, this exponent has different table of symbols for below-mentioned equations.

\section{THE FAILURE CRITERION}

A ccording to the above-mentioned concept, using the maximum stress intensity factor $K_{\max }$ as the controlling parameter $\xi$ and replacing critical time $\tau_{c}$ with the critical hydrogen accumulation peak (maximum local hydrogen concentration) $C_{H \max }$ ahead of the crack tip, the damage evolution equation can be written as

$$
C_{H \max } K_{\max }^{b}=\text { const }
$$

It is assumed that the shape of the loading cycle is not changed. Eq. (8) gives the physical criterion for local fatigue failure in the fracture process zone, i.e. in the vicinity of the fatigue crack tip, and reflects changes in the hydrogen peak which resulted from the hydrogen redistribution due to the increase of the maximum stress intensity factor as the crack length increases under fatigue loading. This conclusion is in agreement with the observed experimental results (Fig. 4) for different values of the maximum stress intensity factor.

Fatigue crack growth behaviour can be described by the following equation [12] 


$$
\frac{d l}{d N}=V^{*}\left(\frac{K_{\max }}{K_{f C}}\right)^{k}=C^{\prime}(1-R)^{k} K_{\max }^{k}
$$

where $V^{*}=C^{\prime}(1-R)^{k} K_{f C}^{k}, C^{\prime}$ and $k$ are constants of the material and loading conditions. The process of local failure in the vicinity of the crack tip has interrupted nature and the crack propagates by means of discrete extension (crack jump) by the value $\Delta a_{i}$. After the jump, further crack propagation requires a certain time (cycle $\Delta N$ of loading) to accumulate fatigue damage and redistribute hydrogen in the vicinity of the crack tip until the condition (8) is reached. Thus, the parameter $V^{*}=\Delta a_{i} / \Delta N$ is connected with the discrete nature of fatigue crack propagation. The value $V^{*}$ slightly decreases for the hydrogen-charged specimens (Tab. 3). This variation is associated with a reduction of the crack increment $\Delta a_{i}$ in the hydrogen-charged specimen rather than with an increase of $\Delta N$. It should be also mentioned that in the presence of a microcracks, the steel with internal hydrogen can be regarded as a system subjected to the effect of an external hydrogen environment. In this case, the localisation of deformation caused by hydrogen can be considered as one of the hydrogen embrittlement mechanisms [17]. It is obvious that more intensive localisation of deformation caused by interaction of hydrogen with moving dislocations and by activation of their sources will be also observed if the steel contains steep hydrogen concentration gradients.

\section{CONCLUSIONS}

7 he effect of hydrogen, which was artificially charged into specimens by a cathodic charging method, on the fracture toughness and fatigue crack growth behaviour in the martensitic high strength steel has been investigated. The distribution of hydrogen concentration in the zone of the fatigue crack tip and at its edges has been analysed by the secondary ion mass spectrometry method. The following conclusions can be drawn from the present study.

The variation of the cathodic current density does not influence on the ratio of the maximum local concentration $C_{H}$ of hydrogen ahead of the crack tip to the volume concentration $C_{0}$ of hydrogen which is determined by the value of the applied stress intensity factor $K_{10}$.

The generalized concept of damage evolution has been employed to describe fatigue crack propagation in connection with the hydrogen redistribution ahead of the crack tip. The failure criterion based on the hydrogen peak in the vicinity of the fatigue crack tip and the maximum stress intensity factor has been proposed. The local concentration of hydrogen in the vicinity of the crack tip is a function of the stress intensity factor, i.e. higher values of $K_{\max }$ lead to the lower hydrogen peak. The criterion explains experimentally observed changes in the hydrogen peak which resulted from the hydrogen redistribution due to the increase of the maximum stress intensity factor as the crack length increases under fatigue loading.

\section{REFERENCES}

[1] S.A. Ahmad, D.A. Ryder, T.A. Davis, Engineering Fracture Mechanics, 7 (1975) 357.

[2] R.L.S. Thomas, J.R. Scully, R.P. Gangloff, Metallurgical and Materials Transactions, 34(A) (2003) 327.

[3] Y. Kim, Y. J. Chao, Marty J. Pechersky, Michael J. Morgan, Int. J. of Fracture, 134 (2005) 339.

[4] J. Capelle, J. Gilgert, I. Dmytrakh, G. Pluvinage, Engineering Fracture Mechanics, 78 (2011) 364.

[5] C.J. McMahon, Engineering Fracture Mechanics, 68 (2001) 773.

[6] A.T. Yokobori, Int. J. of Fracture, 128 (2004) 121.

[7] Y. Oda, H. Noguchi, Int. J. of Fracture, 132 (2005) 99.

[8] Y. Murakami, Int. J. of Fracture, 138 (2006) 167.

[9] I. Dmytrakh, O. Smiyan, A. Syrotyuk, In: Proceedings of the 18th European Conference on Fracture. Fracture of Materials and Structures from Micro to Macro Scale. Dresden, Germany. (2010) 13.

[10] H.W. Liu, Transaction of ASME: J of Basic Engineering, 92 (1070) 633.

[11] S. Wu, L. Chen, M. Liu, Acta Metall. Sinica., 26 (1990) A86.

[12] Yu.G. Matvienko, V. E. Vygovskii, E. N. Lubnin, V. B. Spiridonov, Materials Sciences, 26 (3) (1990) 251. 
[13] H. Gao, W. Cao, C. Fang, E. R. de los Rios, Fatigue and Fracture of Engineering Materials and Structures, 17 (1994) 1213.

[14] Yu.G. Matvienko, In: Integrity of Pipelines Transporting Hydrocarbons, NATO Science for Peace and Security Series C: Environmental Security / eds.: G. Bolzon, T. Boukharouba, G. Gabetta, M. Elboujdaini and M. Mellas, Springer Netherlands: (2011) 227.

[15] A. T. Uesugi, M. Sendoh, and M. Shibata, Strength, Fracture and Complexity, 1 (4) (2003) 187.

[16] N. Saintier, T. Awane, J.M. Olive, S. Matsuoka, Y. Murakami. International journal of Hydrogen Energy, 36 (2011) 8630.

[17] M.R. Louthan, Scripta Metall., 17 (1983) 451.

[18] H. Haken Advanced Synergetic: Instability Hierarchies of Self-Organizing Systems and Devices. Berlin, Heidelberg, New York, Tokyo: Springer-Verlag, (1983). 